\title{
Detection of Meningeosis neoplastica by real-time quantitation of telomerase activity
}

\author{
Gunter Assmann ${ }^{1}$, Christian Werner², Markus \\ Herrmann $^{3, a}$, Dunja Momber ${ }^{1}$, Thomas Krenn ${ }^{4}$, \\ Daniela Lothschütz ${ }^{4}$, Lars Fischer ${ }^{5}$, Sandra \\ Hess $^{1}$, Ralf Ketter ${ }^{6}$, Wolfgang Feiden ${ }^{7}$, Stefan \\ Jung $^{8}$, Wolfgang Herrmann ${ }^{3}$, Jörg Schubert ${ }^{1}$, \\ Michael Pfreundschuh ${ }^{1}$ and Thomas \\ Widmann ${ }^{1, *}$ \\ ${ }^{1}$ Klinik und Poliklinik für Innere Medizin I, \\ Universitätsklinikum Homburg, Homburg/Saar, \\ Germany \\ ${ }^{2}$ Klinik und Poliklinik für Innere Medizin III, \\ Universitätsklinikum Homburg, Homburg/Saar, \\ Germany \\ ${ }^{3}$ Klinische Chemie und Laboratoriumsmedizin, \\ Universitätsklinikum Homburg, Homburg/Saar, \\ Germany \\ ${ }^{4}$ Klinik für Pädiatrische Onkologie und Hämatologie, \\ Universitätsklinikum Homburg, Homburg/Saar, \\ Germany \\ ${ }^{5}$ Medizinische Klinik III, Charité, Berlin \\ ${ }^{6}$ Klinik für Neurochirurgie, Universitätsklinikum \\ Homburg, Homburg/Saar, Germany \\ ${ }^{7}$ Institut für Neuropathologie, Universitätsklinikum \\ Homburg, Homburg/Saar, Germany \\ ${ }^{8}$ Klinik für Neurologie, Caritas Krankenhaus \\ Dillingen, Germany
}

\begin{abstract}
Background: Analysis of cerebrospinal fluid (CSF) to discriminate between benign and malignant conditions is of fundamental importance for the physician and the patient because of the differential therapeutic options and resulting morbidity and mortality. Most human tumours demonstrate increased telomerase activity (TA). Recent technical advances in the detection of TA allow for sensitive and specific detection within $4 \mathrm{~h}$. Thus, the detection of TA is suitable for routine clinical testing.

Methods: This study examines TA in cellular proteins in CSF from 111 patients compared to cytomorphological and laboratory examination.

Results: A positive result for TA in cellular proteins of CSF was correlated significantly with Meningeosis neoplastica, but not with non-malignant conditions.

${ }^{a}$ Current address: ANZAC Research Institute, The University of Sydney, Bone Biology Unit, Sydney, Australia

*Corresponding author: Dr. Thomas Widmann, Institut für Medizinische Mikrobiologie und Hygiene,

Universitätsklinikum Homburg, 66421 Homburg/Saar,

Germany

Phone: +49-6841-1623944, Fax: +49-6841-1623965,

E-mail: Thomas.Widmann@uks.eu

Received April 4, 2009; accepted June 22, 2009;

previously published online September 11, 2009
\end{abstract}

Telomerase was not detected in CSF supernatant, despite positive results in cellular proteins from identical patients. Furthermore, a 48-h time delay during the pre-analytic processing is not critical for detection of TA detection in native CSF when stored at room temperature.

Conclusions: We conclude that TA is a promising marker for the detection of Meningeosis neoplastica and warrants further study.

Clin Chem Lab Med 2009;47:1271-6.

Keywords: cerebrospinal fluid; Meningeosis neoplastica; real-time quantitative telomere repeat amplification protocol; telomerase activity.

\section{Introduction}

Cytological analysis of cerebrospinal fluid (CSF) is the standard procedure for the diagnosis of meningitis or Meningeosis neoplastica. The incidence of Meningeosis neoplastica diagnosed by cytology in a large nonselected series of 5951 specimens by Prayson and Fischler (1) was $5 \%$ in adults and $8 \%$ in paediatric patients. Despite the low incidence of Meningeosis neoplastica, discrimination between non-malignant and malignant cytology is of vital importance for the patient due to fundamentally differential therapeutic regimens (anti-infective, immunosuppressive or no therapy vs. anti-neoplastic therapy). Leptomeningeal involvement is present in about $6 \%$ of patients diagnosed with acute lymphatic leukaemia (2) and develops in about $5 \%$ of patients with solid tumours $(3,4)$.

In spite of substantial advances in prophylaxis and therapy, the detection of Meningeosis neoplastica usually requires an intensification of anti-neoplastic therapy and predicts an unfavourable outcome in patients resulting from increased disease and therapy related morbidity and mortality $(5,6)$. Alternative diagnostic technologies including gadolinium imaging (7) or mass spectroscopy (8) are inferior to CSF cytology. For lymphoid neoplasms, multiparameter flow cytometric analysis of $\operatorname{CSF}(9,10)$ may be helpful as an adjunct to cytology, which remains the gold standard for the diagnosis of Meningeosis neoplastica (11).

The enzyme telomerase consists of a catalytic protein subunit human telomerase reverse transcriptase (hTert) and a ribonucleic acid (RNA) template human telomerase RNA ( $\mathrm{hTr}$ ). Telomerase is capable of telomere repeat elongation in vivo involving a unique reverse transcriptase activity, able to regulate telomere length in vivo (12). However, functionally active telomerase is restricted to embryonic stem cells (13), germ line cells (14), rarely in differentiated cells like 
lymphocytes or adult haematopoietic stem cells (15-17) and, notably, in neoplastic cells. Because telomeres progressively shorten with each cellular division as a sign of replicative senescence (18), stabilisation of telomeres by activation of telomerase is presumed to be a fundamental mechanism for indefinite proliferation in neoplasia. Up to $90 \%$ of human tumours express telomerase (19), whereas the remaining tumours have developed alternative mechanisms to stabilize their telomeres (20).

Here, we exploited the fact that neoplastic cells are characterised by highly active telomerase in order to facilitate a more rapid diagnoses of Meningeosis neoplastica. With the development of a fast and reliable real-time polymerase chain reaction (PCR) based methodology [real-time quantitative telomere repeat amplification protocol (RQ-Trap) assay as reported by Wege et al. (21)] the detection of telomerase activation may be potentially useful in clinical practice. We have previously shown that telomerase activity (TA) can be measured in lymphoblasts from peripheral blood or CSF using the RO-Trap protocol (16). The present study was designed to assess the feasibility and diagnostic accuracy of measuring TA on a routine basis for the detection of Meningeosis neoplastica in diagnostic CSF.

\section{Materials and methods}

The study was approved by the Local Ethics Committee and all study participants gave written informed consent. CSF was drawn from patients undergoing routine lumbar puncture and was centrifuged at $800 \mathrm{~g}$ for $5 \mathrm{~min}$, within $30 \mathrm{~min}$ following collection of CSF. The cell pellet and the supernatant were stored at $-80^{\circ} \mathrm{C}$ until further analysis. Samples for TA assays were extracted following standard protocols (22). Cytological examination was performed by an expert cytopathologist who was not aware of other laboratory test results.

\section{Telomerase activity}

TA was quantified under real-time PCR conditions utilizing the RQ-Trap procedure (Lightcycler instrument, Roche, Mannheim, Germany) as reported by Wege et al. (21) with minor modifications. In brief, duplicates of protein extracts from 1000 cells or CSF supernatant were mixed with $0.1 \mu \mathrm{g}$ of Primer TS (template) and $0.05 \mu \mathrm{g}$ Primer ACX (23) in $20 \mu \mathrm{L}$ Lightcycler Fast Start SYBR Green PCR Master Mix (Roche, Mannheim, Germany) containing $1.5 \mathrm{mM} \mathrm{MgCl}_{2}$. The mixture was incubated at $30^{\circ} \mathrm{C}$ for $30 \mathrm{~min}$ to allow template elongation by TA. Immediately following transfer to the Lightcycler instrument, telomerase was heat-inactivated and hotstart DNA polymerase activated by incubation at $95^{\circ} \mathrm{C}$ for $10 \mathrm{~min}$. Thirty-six cycles of amplification were performed with $20 \mathrm{~s}$ denaturation at $95^{\circ} \mathrm{C}$, annealing for $30 \mathrm{~s}$ at $60^{\circ} \mathrm{C}$, and elongation for $50 \mathrm{~s}$ at $72^{\circ} \mathrm{C}$. TA was displayed as relative TA to a standard of 1000 HEK293 cells. A standard titration curve of HEK293 cells was established using $0-1000$ cells to ensure linearity of the assay. A positive signal was obtained with a minimal dilution of $8 / 1000$ HEK293 cells in repetitive experiments. A positive result of the RQ-Trap assay was assumed when the sample's $C_{t}$ value was three standard deviations (SD) above the mean $C_{t}$ value obtained with repetitive measures of lysed and heat inactivated HEK293 cells. Therefore, the cut-off for a positive test result was defined as a relative TA of $1 \%$ (equally to a TA of $10 / 1000$ HEK293 cells). Heat inactivated lysate from 1000 HEK293 cells served as a negative control and telomerase positive CD19 + lymphoblasts (16) served as the positive control. Quantitative levels of TA were obtained from cellular proteins of 1000 cells, serial dilutions of cellular proteins or supernatant.

\section{Tissue culture}

Cell lines (Jurkat, Daudi, Karpas, K562, L540 and HEK293) were purchased from ATCC (Wesel, Germany) and grown in RPMI 1640 (supplemented with 10\% fetal calf serum, glutamine and penicillin and streptomycin, all from Gibco, Karlsruhe, Germany). Lines were maintained in a $37^{\circ} \mathrm{C}, 5 \% \mathrm{CO}_{2}$ atmosphere and divided splitted twice per week.

\section{Statistical analyses}

Graphs and statistical analyses were performed using SigmaPlot (Version 7.0) and SigmaStat (Version 2.03; both from SPSS Inc, Chicago, IL, USA). Repeated measure ANOVA was used for the analysis of TA at different time points during the time delay experiment. Differences in TA from cellular proteins or tissue culture supernatant were analysed using the unpaired Student's t-test. Correlation between (telomerase-) elongated template and cycle number was determined with linear regression analysis.

\section{Results}

\section{TA determined by RQ-Trap assay}

Serial dilutions of HEK293 cells were assayed for TA to generate a standard curve for comparison with other cell lines and clinical samples. The RQ-Trap assay showed reproducible linearity from 1000 to 8 HEK293 cells [(Figure 1: $R^{2}=0.99$, Figure 2 see online Supplementary data)].

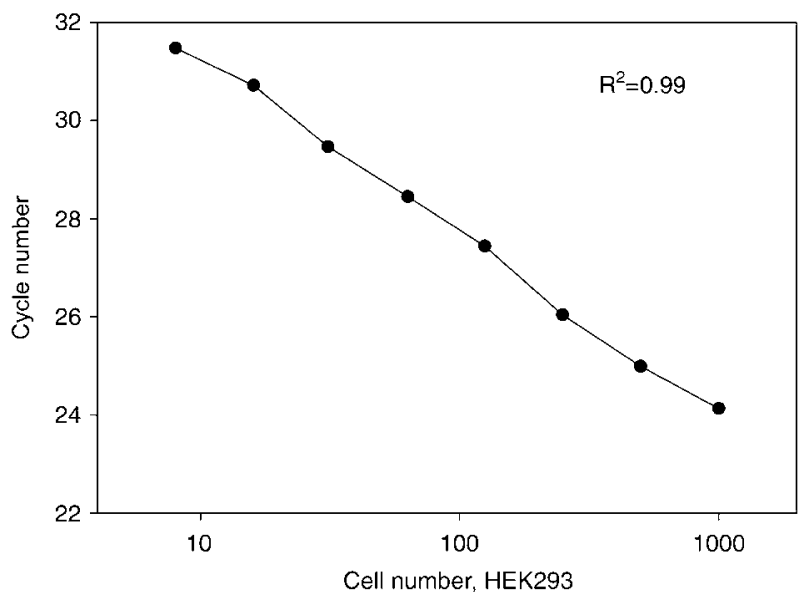

Figure 1 Correlation of telomerase activity (TA) to cell number in serially diluted HEK293 cells.

There was a linear correlation between detection of TA and the HEK293 cell number in the range of 1000-8 HEK293 cells $\left(R^{2}=0.99\right)$. 


\section{TA in Meningeosis neoplastica}

To test our hypothesis that the RQ-Trap assay is appropriate for clinical usage in the detection of Meningeosis neoplastica, we have studied 111 patients during routine clinical CSF examination. Diagnosis of Meningeosis neoplastica was established cytologically in 15 cases by a local neuropathologist (Table 1). All of these specimens had detectable TA. A negative result in the RQ-Trap assay correlated with non-malignant CSF in 96 patients. However, one patient had a positive result without clinical signs of Meningeosis neoplastica. This patient was a 6-year-old male patient who was in clinical remission with T-cell non-Hodgkin's lymphoma (NHL) and undergoing lumbar puncture for prophylactic intrathecal methotrexate chemotherapy. Routinely obtained CSF of this asymptomatic patient showed an increased cell number $(23 / \mu \mathrm{L}$, upper normal value $3 / \mu L)$ in combination with increased total protein ( $2.53 \mathrm{~g} / \mathrm{L}$, upper normal value $0.45 \mathrm{~g} / \mathrm{L}$ ). There was no clinical correlation to an infection or malignant condition. Interestingly, the patient had received prophylactic intrathecal chemotherapy 2 weeks prior to lumbar puncture. The patient never developed meningeal involvement of his T-cell NHL and remains in continuous complete remission. The sensitivity to detect Meningeosis neoplastica with the RQ-Trap assay was $100 \%$ (15/15 samples), whereas the specificity was $98.9 \%$ (95/96 samples). This resulted in a positive predictive value (positive result for TA) of 0.94 and a negative predictive value of 1.0 for the diagnosis Meningeosis neoplastica (Table 2). Furthermore, there was a highly significant association between quantitative values of TA and a diagnosis of Meningeosis neoplastica (Figure 3, $\mathrm{p}<0.001$ ).

\section{TA in supernatants}

In order to potentially ease and more rapidly process CSF specimens, we aimed to quantify TA in CSF supernatant. TA was measured in eight patients with Meningeosis neoplastica as well as from tissue culture supernatants from different cell lines $(n=5)$. None of the patients with clinically diagnosed Meningeosis neoplastica had detectable TA in their CSF supernatant, despite traceable TA in corresponding CSF cellular proteins (data not shown). In contrast, $4 / 5$ tissue culture supernatants (Karpas, Jurkat, Daudi, K562) showed detectable TA, proving that TA can be measured directly in the supernatant (Figure 4).

\section{Telomerase stability during the pre-analytic process}

Finally, we examined the potential limitations of the handling process of diagnostic CSF samples. Inactivation of telomerase during any time delay in the pre-analytic process may compromise the assay sensitivity resulting in false negative results. We attempted to mimic delays in processing by incubating HEK293 cells or their protein extracts from $0 \mathrm{~h}$ to $48 \mathrm{~h}$ at room temperature before measuring TA (Figure 5). Stable TA levels were documented when whole cells were incubated at room temperature, whereas protein isolation extraction followed by incubation at room temperature led to rapidly decreasing TA levels $(p<0.001)$.

\section{Discussion}

Meningeosis neoplastica is one of the most deleterious complications of neoplastic diseases requiring a prompt and reliable diagnosis. To the best of our knowledge, this is the first report to correlate TA measured by RQ-Trap to the cytopathological diagnosis of Meningeosis neoplastica in a routine clinical setting.

The test protocol requires only a small amount of CSF (250 $\mu \mathrm{L}$ for CSF pleocytosis, depending on the cell number) which can be obtained during routine laboratory evaluation. The baseline sensitivity level of 8 malignant cells $/ 1000$ cells that we found is comparable to a recent report of Jakupciak et al. (24), who reported a positive result of TA in 10/1000 A594 cells with the RQ-Trap protocol. Notably, the RQ-Trap protocol was about 10 times more sensitive compared to an hTert mRNA quantification in the same cells. Therefore, it will be of interest in future studies to correlate results of TA to markers for minimal residual disease in selected patients. With the rapid and reliable assay presented in this study, 15/15 patients with Meningeosis neoplastica were identified correctly. Furthermore, considering the high negative predictive value of 1.0 , this assay may serve as a screening test for menigeosis neoplastica. However, the results of this pilot study are preliminary due to the limited patient number and require confirmation using a larger population. Kleinschmidt-DeMasters et al. (25) reported a sensitivity of $60 \%$ and specificity of $87 \%$ for the detection of Meningeosis neoplastica with assessment of TA using conventional TRAP. The increased sensitivity and specificity in our study may be due to improvements in the assay system for the detection of TA. Compared to conventional TA detection methods that rely on end time PCR (e.g., PCRELISA or gel detection systems), the RQ-Trap assay provides a greater linear range of analysis that is more sensitive when compared to commercially available PCR-ELISA applications (21). Moreover, with the development of more specific primers to reduce primer-dimers, the RQ-Trap provides a higher specificity. Finally, using RQ-Trap, the total analysis time is reduced to $<4 \mathrm{~h}$ with hands-on time $<20 \mathrm{~min}$. Also, post-PCR carryover sample analysis errors are largely reduced due to the closed tube application.

Our results show a correlation of TA with the presence of malignancy in CSF and are comparable to studies using other body fluids (26-28). This indicates that TA may be an easy to measure marker of malignant cells from a broad spectrum of routine diagnostic samples. However, there are limitations in sensitivity and specificity depending on the sample source. Attempts to detect TA in the CSF supernatant failed for patients' samples. However, tissue culture 


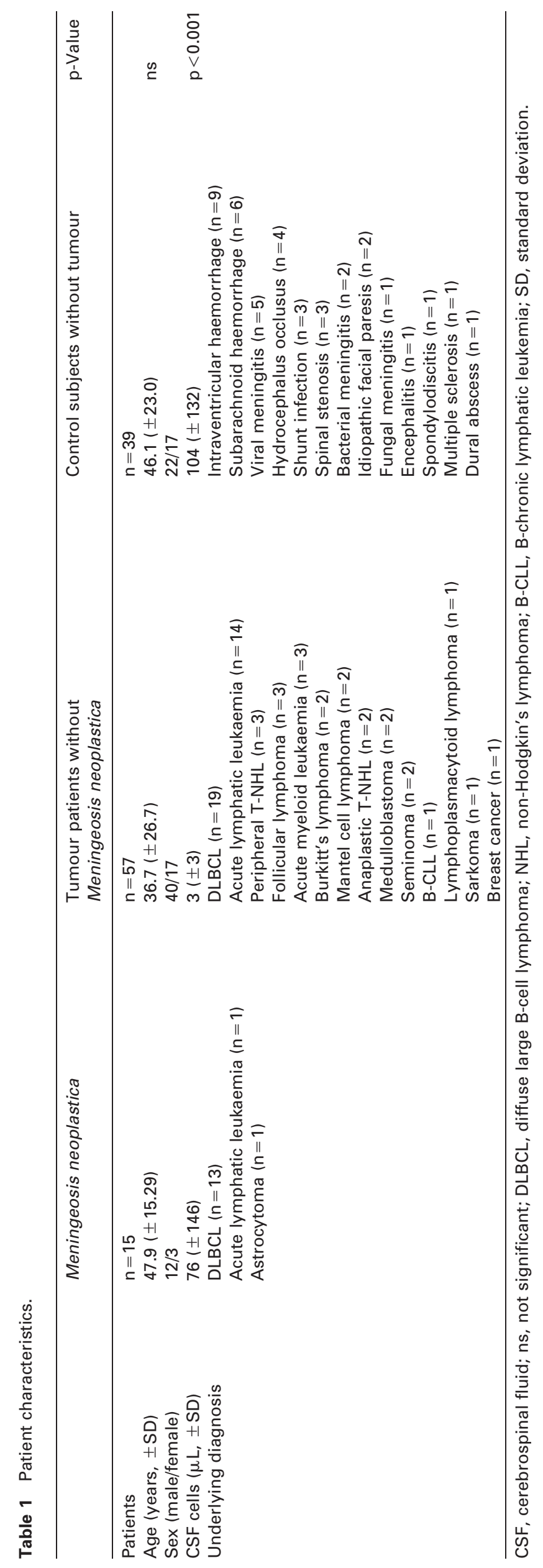


Table 2 Contingency table.

\begin{tabular}{lcc}
\hline & $\begin{array}{l}\text { Meningeosis } \\
\text { neoplastica, } \mathrm{n}\end{array}$ & $\begin{array}{l}\text { No } \\
\text { Meningeosis } \\
\text { neoplastica, } \mathrm{n}\end{array}$ \\
\hline Telomerase activity, $\mathrm{n}$ & 15 & 1 \\
No telomerase activity, $\mathrm{n}$ & 0 & 95 \\
Sum, $\mathrm{n}$ & 15 & 96 \\
\hline
\end{tabular}

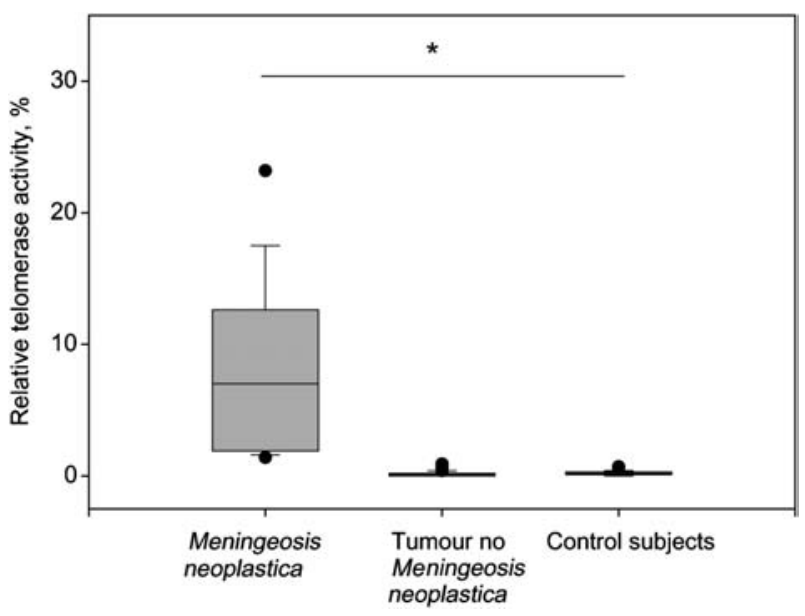

Figure 3 Relative telomerase activity (TA) in Meningeosis neoplastica and from non-neoplastic CSF.

The level of TA is displayed in \% as relative TA compared to that of 1000 HEK293 cells and expressed as box plots, displaying the median as horizontal lines and boxes as 25th and 75th percentile as well as whiskers indicating 10th and 90th percentile. The real-time quantitative telomere repeat amplification protocol (RO-Trap) assay for relative TA discriminated Meningeosis neoplastica from non-malignant conditions $\left({ }^{*} p<0.001\right)$.

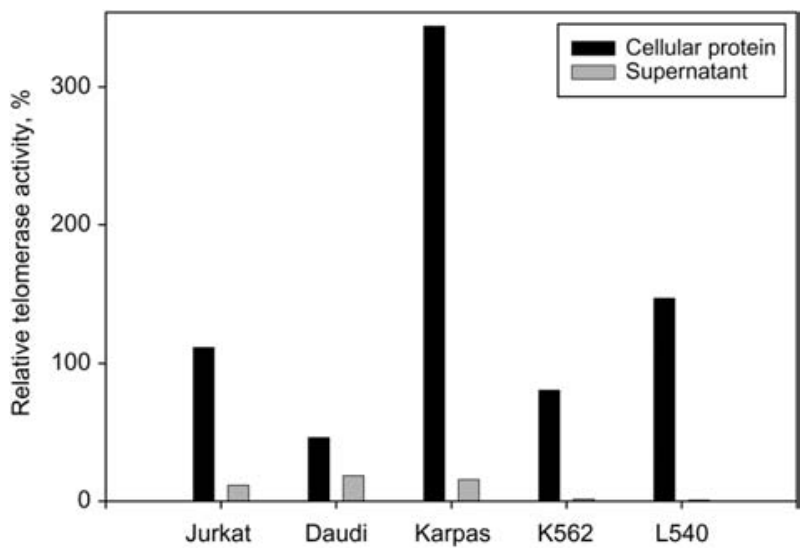

Figure 4 Telomerase activity (TA) in cellular proteins or tissue culture supernatant.

Relative TA was quantified in cellular proteins ( 1000 cells) of 5 different malignant cell lines (Jurkat, Daudi, Karpas, K562 and L540) as well as in tissue culture supernatant. TA in tissue culture supernatant was detectable in $4 / 5$ cell lines at lower levels compared to TA from cellular protein $(p=0.03)$.

supernatants of malignant cell lines showed reproducibly increased levels of TA. This suggests that TA in CSF supernatant may either be diluted by the physiological CSF circulation (29), or our assay system

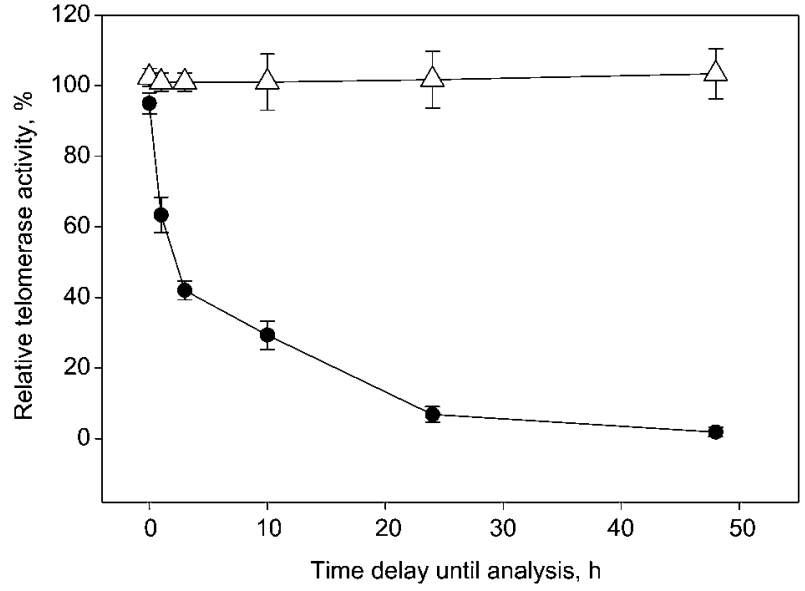

Figure 5 Stability of telomerase activity (TA).

TA measured from HEK293 cells during an incubation period of up to $48 \mathrm{~h}$ at room temperature. White triangles show stable TA in cellular proteins that were freshly isolated after $0 \mathrm{~h}, 1 \mathrm{~h}, 3 \mathrm{~h}, 10 \mathrm{~h}, 24 \mathrm{~h}$ and $48 \mathrm{~h}(\mathrm{p}=0.89)$. In contrast, TA in isolated proteins (protein isolation at $0 \mathrm{~h}$ ) stored at room temperature for $48 \mathrm{~h}$ declined rapidly $(\mathrm{p}<0.001)$ during the incubation period. All values are expressed as mean \pm SEM.

may not be sensitive enough to detect minimal amounts of TA in CSF. Furthermore, extracellular TA may be rapidly inactivated in a time dependent manner if samples are not analysed immediately. This was illustrated by the time-dependent decrease in TA from protein extracts over time. Despite this, TA is readily detectable in tumour cells from CSF for at least $48 \mathrm{~h}$ at room temperature.

In conclusion, we demonstrate a fast, reliable and robust method for the detection of TA in a clinical pilot study for the detection of Meningeosis neoplastica. Conceivably, RQ-Trap may function as a "CSF quick test" to identify high-risk patients with leptomeningeal tumour involvement and may, thus, supplement standard cytomorphological examination. The assay is feasible for routine clinical usage and, therefore, warrants further study in order to assess the precise role of TA in CSF, as well as in the context of repetitive testing during anti-tumour therapy.

Supplementary data associated with this article can be found in the online version at: http://www.reference-global.com/ doi/suppl/10.1515/CCLM.2009.258.

\section{Acknowledgements}

We thank all participating patients for donating CSF and Natalie Fadle for excellent assistance during cell culture experiments. Thomas Widmann was supported by institutional funding.

\section{References}

1. Prayson RA, Fischler DF. Cerebrospinal fluid cytology: an 11-year experience with 5951 specimens. Arch Pathol Lab Med 1998;122:47-51.

2. Gokbuget N, Hoelzer D. Meningeosis leukaemica in adult acute lymphoblastic leukaemia. J Neurooncol 1998;38: 167-80. 
3. Bleyer WA, Byrne TN. Leptomeningeal cancer in leukemia and solid tumors. Curr Probl Cancer 1988;12:181238.

4. Chamberlain MC. Leptomeningeal metastases: a review of evaluation and treatment. J Neurooncol 1998;37: 271-84.

5. Cole BF, Glantz MJ, Jaeckle KA, Chamberlain MC, Mackowiak JI. Quality-of-life-adjusted survival comparison of sustained-release cytosine arabinoside versus intrathecal methotrexate for treatment of solid tumor neoplastic meningitis. Cancer 2003;97:3053-60.

6. Jabbour E, O'Brien S, Kantarjian H, Garcia-Manero G, Ferrajoli A, Ravandi F, et al. Neurologic complications associated with intrathecal liposomal cytarabine given prophylactically in combination with high-dose methotrexate and cytarabine to patients with acute lymphocytic leukemia. Blood 2007;109:3214-8.

7. Straathof CS, de Bruin HG, Dippel DW, Vecht CJ. The diagnostic accuracy of magnetic resonance imaging and cerebrospinal fluid cytology in leptomeningeal metastasis. J Neurol 1999;246:810-4

8. Dekker LJ, Boogerd W, Stockhammer G, Dalebout JC, Siccama I, Zheng $P$, et al. MALDI-TOF mass spectrometry analysis of cerebrospinal fluid tryptic peptide profiles to diagnose leptomeningeal metastases in patients with breast cancer. Mol Cell Proteomics 2005;4:1341-9.

9. Schinstine M, Filie AC, Wilson W, Stetler-Stevenson M, Abati A. Detection of malignant hematopoietic cells in cerebral spinal fluid previously diagnosed as atypical or suspicious. Cancer 2006;108:157-62.

10. Kraan J, Gratama JW, Haioun C, Orfao A, Plonquet A, Porwit $A$, et al. Flow cytometric immunophenotyping of cerebrospinal fluid. Curr Protoc Cytom 2008; Chapter 6:Unit 6.25

11. Glantz MJ, Cole BF, Glantz LK, Cobb J, Mills P, Lekos A, et al. Cerebrospinal fluid cytology in patients with cancer: minimizing false-negative results. Cancer 1998;82: 733-9.

12. Greider CW, Blackburn EH. Identification of a specific telomere terminal transferase activity in Tetrahymena extracts. Cell 1985;43:405-13.

13. Thomson JA, Itskovitz-Eldor J, Shapiro SS, Waknitz MA, Swiergiel JJ, Marshall VS, et al. Embryonic stem cell lines derived from human blastocysts. Science 1998;282: 1145-7.

14. Schonland SO, Widmann T, Lopez-Benitez CM, Zimmer J, Bryl E, Goronzy JJ, et al. Premature telomeric loss in rheumatoid arthritis is genetically determined and involves both myeloid and lymphoid cell lineages. Proc Natl Acad Sci USA 2003;100:13471-6.
15. Widmann TA, Willmann B, Pfreundschuh M, Beelen DW. Influence of telomere length on short-term recovery after allogeneic stem cell transplantation. Exp Hematol 2005;33:1257-61.

16. Widmann TA, Herrmann M, Taha N, Konig J, Pfreundschuh M. Short telomeres in aggressive non-Hodgkin's lymphoma as a risk factor in lymphomagenesis. Exp Hematol 2007;35:939-46.

17. Yui J, Chiu CP, Lansdorp PM. Telomerase activity in candidate stem cells from fetal liver and adult bone marrow. Blood 1998;91:3255-62.

18. Hayflick L. Human cells and aging. Sci Am 1968;218: 32-7.

19. Hiyama E, Hiyama K. Telomerase as tumor marker. Cancer Lett 2003;194:221-33.

20. Henson JD, Neumann AA, Yeager TR, Reddel RR. Alternative lengthening of telomeres in mammalian cells. Oncogene 2002;21:598-610.

21. Wege H, Chui MS, Le HT, Tran JM, Zern MA. SYBR Green real-time telomeric repeat amplification protocol for the rapid quantification of telomerase activity. Nucleic Acids Res 2003;31:E3.

22. Kim NW, Piatyszek MA, Prowse KR, Harley CB, West MD, Ho PL, et al. Specific association of human telomerase activity with immortal cells and cancer. Science 1994; 266:2011-5

23. Kim NW, Wu F. Advances in quantification and characterization of telomerase activity by the telomeric repeat amplification protocol (TRAP). Nucleic Acids Res 1997; 25:2595-7.

24. Jakupciak JP, Barker PE, Wang W, Srivastava S, Atha $\mathrm{DH}$. Preparation and characterization of candidate reference materials for telomerase assays. Clin Chem 2005; 51:1443-50.

25. Kleinschmidt-DeMasters BK, Evans LC, Bitter MA, Shroyer AL, Shroyer KR. Part II. Telomerase expression in cerebrospinal fluid specimens as an adjunct to cytologic diagnosis. J Neurol Sci 1998;161:124-34.

26. Sanchini MA, Gunelli R, Nanni O, Bravaccini S, Fabbri C, Sermasi A, et al. Relevance of urine telomerase in the diagnosis of bladder cancer. J Am Med Assoc 2005; 294:2052-6.

27. Nieh S, Chen SF, Fu E, Jan Cl, Lee WH. Detection of the human telomerase RNA component by in situ hybridization in cells from body fluids. Acta Cytol 2005;49:31-7.

28. Hess JL, Highsmith WE Jr. Telomerase detection in body fluids. Clin Chem 2002;48:18-24.

29. Brodbelt A, Stoodley M. CSF pathways: a review. $\mathrm{Br} \mathrm{J}$ Neurosurg 2007;21:510-20. 